PROCEEDINGS OF THE

AMERICAN MATHEMATICAL SOCIETY

Volume 126, Number 2, February 1998, Pages 453-460

S $0002-9939(98) 04145-8$

\title{
PROPERTIES OF SUBGENERATORS OF $C$-REGULARIZED SEMIGROUPS
}

\author{
SHENG WANG WANG
}

(Communicated by Palle E. T. Jorgensen)

\begin{abstract}
We introduce two operations $\wedge, \vee$ in the set $\mathcal{G}$ of subgenerators of a given $C$ - regularized semigroup and prove that $\mathcal{G}$ is a complete partially ordered lattice with respect to $\wedge, \vee$ and the operator inclusion $\subseteq$. Also presented are some other properties and examples for $\mathcal{G}$.
\end{abstract}

\section{INTRODUCTION}

In dealing with the many physical problems that may be modeled as an abstract Cauchy problem

$$
\frac{d}{d t} u(t, x)=A u(t, x)(t \geq 0), u(0, x)=x,
$$

where $A$ is a closed linear operator on a Banach space $X$, and $u(\cdot, x) \in \mathbf{C}([0, \infty), X)$, well-posedness correponds to $A$ generating a strongly continuous semigroup. When (ACP) is not well-posed, a useful concept for dealing with it is a $C$-regularized semigroup (Definition 1.1). When $A$ generates a $C$-regularized semigroup, then (ACP) has a unique mild solution, for all $x$ in the image of $C$, a unique strong solution, for all $x \in C(D(A))$, and $u\left(t, x_{n}\right) \longrightarrow u(t, x)$, uniformly for $t$ in every compact subset of $[0, \infty)$, whenever $C^{-1} x_{n} \longrightarrow C^{-1} x$.

However, in order that (ACP) have all these solutions, it is not necessary that $A$ generates a $C$-regularized semigroup; it is only necessary that $A$ be a subgenerator (Definition 1.2) of a $C$-regularized semigroup. This was first observed only very recently, in [7, Counter example 0.2]. In fact, (ACP) has a unique mild solution for all $x \in \operatorname{Im}(C)$ if and only if $A$ is a subgenerator of a $C$-regularized semigroup [7, Theorem 3.3].

Thus from the point of view of applications, there is no difference between a generator and a subgenerator. In practice, verifying that $A$ is a subgenerator of a $C$-regularized semigroup is much easier than showing that $A$ itself is the generator. Hence, the subgenerators become the objects of interest. Unfortunately, up to now the properties of the set of subgenerators of a $C$-regularized semigroup are not clear. This paper attempts to study these and presents several results and examples.

Throughout $X$ is a Banach space and $L(X)$ is the algebra of all bounded linear operators on $X$. Let $C \in L(X)$.

Received by the editors December 14, 1995 and, in revised form, April 19, 1996 and August 8, 1996.

1991 Mathematics Subject Classification. Primary 47D05, 47D06, 47F05.

(C)1998 American Mathematical Society 
Definition 1.1 ([3], [4], [6], [7], [8]). The strongly continuous family of operators

$$
\{W(t)\}_{t \geq 0} \subset L(X)
$$

is a $C$-regularized semigroup if it satisfies:

(1) $W(0)=C$, and

(2) $W(t) W(s)=C W(t+s)$, for all $t, s \geq 0$.

$\{W(t)\}_{t \geq 0}$ is nondegenerate if $W(t) x \equiv 0$, for all $t \geq 0$, implies $x=0$.

In [7, Proposition 2.2] it is shown that $\{W(t)\}_{t \geq 0}$ is nondegenerate if and only if $C$ is injective. In this paper, we assume that $\{W(t)\}_{t \geq 0}$ is nondegenerate.

Definition 1.2 ([7], [12]). Assume $B$ is closed. We say that $B$ is a subgenerator of the $C$-regularized semigroup $\{W(t)\}_{t \geq 0}$ if

(1) $W(t) B \subseteq B W(t)$ for all $t \geq 0$, and

(2) $\int_{0}^{t} W(s) x d s \in D(B)$ and $B \int_{0}^{t} W(s) x d s=W(t) x-C x \quad \forall x \in X, t \geq 0$.

We also say that $B$ has a $C$-regularized semigroup $\{W(t)\}_{t \geq 0}$ or $\{W(t)\}_{t \geq 0}$ is a $C$-regularized semigroup for $B$.

For convenience, we will use the term subgenerator.

Generally, subgenerators of a given $C$-regularized semigroup are not unique (see Examples 2.13, 2.14 ). However, it is shown in [7, Proposition 2.9] that a $C$-regularized semigroup is uniquely determined by one of its subgenerators.

Definition $1.3([7])$. Assume $\{W(t)\}_{t \geq 0}$ is a $C$-regularized semigroup. Let $D(\tilde{A})$ be the set of all $x \in X$ such that there exists $y \in X$ satisfying

$$
W(t) x-C x=\int_{0}^{t} W(s) y d s \quad \forall t \geq 0 .
$$

Then $\tilde{A} x=y . \tilde{A}$ is called the generator of $\{W(t)\}_{t \geq 0}$.

An analogous definition of the generator of an integrated semigroup appears in [1], [14]. Moreover, it has been proved in [7, Proposition 2.6] that

(1) if $\tilde{A}$ is the generator of $\{W(t)\}_{t \geq 0}$ then $\tilde{A}$ is a subgenerator of $\{W(t)\}_{t \geq 0}$;

(2) $\tilde{A} x=C^{-1} \lim _{t \longrightarrow 0+} \frac{1}{t}(W(t) x-C x)$, with maximal domain.

\section{Properties of Subgenerators}

In this section, we are devoted to the study of properties of subgenerators. Let $\mathcal{G}$ be the set of all subgenerators of the $C$-regularized semigroup $\{W(t)\}_{t \geq 0}$.

Definition 2.1 ([12]). Let $A$ be the operator defined by

$$
\begin{gathered}
D(A)=\left\{\sum_{k=1}^{m} \int_{0}^{t_{k}} W(s) x_{k} d s: x_{k} \in X, t_{k} \geq 0, k=1, \ldots, m\right\} ; \\
A\left[\sum_{k=1}^{m} \int_{0}^{t_{k}} W(s) x_{k} d s\right]=\sum_{k=1}^{m}\left[W\left(t_{k}\right) x_{k}-C x_{k}\right] .
\end{gathered}
$$

Proposition $2.2([7],[12])$. Assume $\{W(t)\}_{t \geq 0}$ is a C-regularized semigroup. Then

(1) A in Definition 2.1 is well-defined and closable;

(2) $\bar{A}$, the closure of $A$, and $\tilde{A}$ are respectively the smallest and largest elements in $\mathcal{G}$, that is, every $B \in \mathcal{G}$ satisfies $\bar{A} \subseteq B \subseteq \tilde{A}$;

(3) for every $B \in \mathcal{G}, C^{-1} B C=\tilde{A}$. 
The following lemma is clear.

Lemma 2.3. For every $T \in L(X)$ and every closable $S, T S \subseteq S T$ implies $T \bar{S} \subseteq$ $\bar{S} T$, where $\bar{S}$ is the closure of $S$.

Lemma 2.4. For any $B_{1}, B_{2} \in \mathcal{G}$, the following hold:

(1) if $x \in D\left(B_{1}\right) \cap D\left(B_{2}\right)$ then $B_{1} x=B_{2} x$;

(2) let $B$ be the operator

$$
D(B)=D\left(B_{1}\right) \cap D\left(B_{2}\right), B x=B_{1} x \quad \forall x \in D(B) .
$$

Then $B$ is closed and $B \in \mathcal{G}$.

Proof. (1) is obvious. As for (2), from Proposition 2.2(2), $\bar{A} \subseteq B \subseteq \tilde{A}$. This, together with the obvious inclusion $W(t) B \subseteq B W(t)$ for all $t \geq 0$, gives $B \in \mathcal{G}$.

Lemma 2.5. For any $B_{1}, B_{2} \in \mathcal{G}$, let $B^{0}$ be the operator

$$
\begin{gathered}
D\left(B^{0}\right)=\operatorname{span}\left[D\left(B_{1}\right) \cup D\left(B_{2}\right)\right] ; \\
B^{0}\left(a_{1} x_{1}+a_{2} x_{2}\right)=a_{1} B_{1} x_{1}+a_{2} B_{2} x_{2} \quad \forall x_{i} \in D\left(B_{i}\right), \quad a_{i} \in \mathbf{C}, i=1,2 .
\end{gathered}
$$

Then $B^{0}$ is closable and its closure $\tilde{B} \in \mathcal{G}$.

Proof. $B^{0}$ is clearly the restriction of $\tilde{A}$ to $\operatorname{span}\left[D\left(B_{1}\right) \cup D\left(B_{2}\right)\right]$, it is well-defined and closable. The inclusions $\bar{A} \subseteq B^{0} \subseteq \tilde{A}$ imply $\bar{A} \subseteq \tilde{B} \subseteq \tilde{A}$. From Lemma 2.3, $W(t) \tilde{B} \subseteq \tilde{B} W(t)$ for all $t \geq 0$. Hence $\tilde{B} \in \mathcal{G}$.

Definition 2.6. For any $B_{1}, B_{2} \in \mathcal{G}$, define $B=B_{1} \wedge B_{2}, \tilde{B}=B_{1} \vee B_{2}$, where $B, \tilde{B}$ are operators defined in Lemmas 2.4 and 2.5, respectively.

Proposition 2.7. With respect to the operations $\wedge, \vee$ and the operator inclusion $\subseteq, \mathcal{G}$ is a complete partially ordered lattice.

Proof. It suffices to claim that $\mathcal{G}$ is complete with respect to $\wedge, \vee$, since the fact that $\mathcal{G}$ is partially ordered with respect to the operator inclusion $\subseteq$ is clear.

For a family $\left\{B_{\alpha}\right\}_{\alpha \in \mathcal{A}} \subseteq \mathcal{G}$, define $B$ to be the operator

$$
\begin{gathered}
D(B)=\bigcap_{\alpha \in \mathcal{A}} D\left(B_{\alpha}\right) ; \\
B x=B_{\alpha} x \quad \forall x \in D(B) \text { and } \alpha \in \mathcal{A} .
\end{gathered}
$$

Define $\tilde{B}$ to be the closure of the following operator:

$$
\begin{gathered}
D\left(B^{0}\right)=\operatorname{span} \bigcup_{\alpha \in \mathcal{A}} D\left(B_{\alpha}\right) \\
B^{0}\left(a_{1} x_{\alpha_{1}}+\ldots+a_{k} x_{\alpha_{k}}\right)=a_{1} B_{\alpha_{1}} x_{\alpha_{1}}+\ldots+a_{k} B_{\alpha_{k}} x_{\alpha_{k}},
\end{gathered}
$$

where $x_{\alpha_{j}} \in D\left(B_{\alpha_{j}}\right), a_{j} \in \mathbf{C}$ for $j=1,2, \ldots, k$. Then $B, \tilde{B} \in \mathcal{G}$ are the lower bound and upper bound of $\left\{B_{\alpha}\right\}_{\alpha \in \mathcal{A}}$, respectively. $\mathcal{G}$ is thus a partially ordered lattice.

Proposition 2.8. Assume $\operatorname{Im}(C)$ is dense in $X$. Then the following hold.

(1) $\bar{A}$ equals the closure of $\tilde{A}$ restricted to $C(D(\tilde{A}))$.

(2) $\mathcal{G}$ is a singleton if and only if $C(D(\tilde{A}))$ is a core for $\tilde{A}$. 
Proof. (1) follows from [7, Proposition 2.6(3) and Theorem 3.3(h)].

(2). From (1), $\mathcal{G}$ is a singleton if and only if $\tilde{A}$ is the closure of itself restricted to $C(D(\tilde{A}))$ if and only if $C(D(\tilde{A}))$ is a core for $\tilde{A}$.

Corollary 2.9. If $\tilde{A}$ is densely defined and $C(D(\tilde{A}))$ is a core for $\tilde{A}$, then $\mathcal{G}$ consists of only the element $\tilde{A}$.

Proof. Since $C(D(\tilde{A}))$ is a core for $\tilde{A}$, it is dense in $D(\tilde{A})$. By the density of $D(\tilde{A})$, $C(D(\tilde{A}))$ is dense in $X$. Now the corollary follows from Proposition 2.8.

Proposition 2.10. $\mathcal{G}$ is totally ordered if and only if $\mathcal{G}$ contains at most two elements.

Proof. "Only if". Suppose that $\mathcal{G}$ contains at least three elements $A_{1}, A_{2}, A_{3}$ satisfying

$$
A_{1} \subsetneq A_{2} \subsetneq A_{3} .
$$

Let $x_{0} \in D\left(A_{3}\right) \backslash D\left(A_{2}\right)$. Define

$$
\begin{gathered}
D\left(A_{2}^{\prime}\right)=D\left(A_{1}\right)+\left\{a x_{0}\right\}, \quad a \in \mathbf{C} ; \\
A_{2}^{\prime}\left(y+a x_{0}\right)=A_{1} y+a A_{3} x_{0} \quad \forall y \in D\left(A_{1}\right), a \in \mathbf{C} .
\end{gathered}
$$

Then $A_{2}^{\prime}$ is well-defined. We now claim that $A_{2}^{\prime}$ is closed. Assume $y_{n}+a_{n} x_{0} \longrightarrow x$ and $A_{2}^{\prime}\left(y_{n}+a_{n} x_{0}\right) \longrightarrow z$, as $n \longrightarrow \infty$. Then $\left\{a_{n}\right\}$ is bounded. Otherwise we may assume $a_{n} \longrightarrow \infty$. Then $\left(y_{n}+a_{n} x_{0}\right) / a_{n} \longrightarrow 0$, hence $y_{n} / a_{n} \longrightarrow-x_{0}$. Since

$$
A_{1}\left(y_{n} / a_{n}\right)=A_{2}^{\prime}\left(y_{n}+a_{n} x_{0}\right) / a_{n}-A_{3} x_{0} \longrightarrow-A_{3} x_{0}
$$

and $A_{1}$ is closed, we have $x_{0} \in D\left(A_{1}\right)$, contradicting the fact that $x_{0} \notin D\left(A_{1}\right)$. Thus we may assume $a_{n} \longrightarrow a_{0}$, as $n \longrightarrow \infty$. From

$$
\begin{gathered}
y_{n} \longrightarrow x-a_{0} x_{0}, \text { and } \\
A_{1} y_{n}=A_{2}^{\prime}\left(y_{n}+a_{n} x_{0}\right)-a_{n} A_{3} x_{0} \longrightarrow z-a_{0} A_{3} x_{0},
\end{gathered}
$$

we have $x-a_{0} x_{0} \in D\left(A_{1}\right)$ and

$$
A_{1}\left(x-a_{0} x_{0}\right)=z-a_{0} A_{3} x_{0} .
$$

This implies

$$
\begin{gathered}
x=\left(x-a_{0} x_{0}\right)+a_{0} x_{0} \in D\left(A_{2}^{\prime}\right), \quad \text { and } \\
A_{2}^{\prime} x=A_{1}\left(x-a_{0} x_{0}\right)+a_{0} A_{3} x_{0}=z .
\end{gathered}
$$

$A_{2}^{\prime}$ is closed.

Next, we prove that

$$
W(t) A_{2}^{\prime} \subseteq A_{2}^{\prime} W(t) \quad \forall t \geq 0 .
$$

For $x \in D\left(A_{3}\right)$, differentiate both sides of

$$
A_{1} \int_{0}^{t} W(s) x d s=\int_{0}^{t} W(s) A_{3} x d s
$$

to obtain

$$
A_{1} W(t) x=W(t) A_{3} x
$$


by the closedness of $A_{1}$. For the previous $y$ and $x_{0},(2.2)$ implies

$$
W(t) A_{2}^{\prime}\left(y+a x_{0}\right)=W(t) A_{3}\left(y+a x_{0}\right)=A_{1} W(t)\left(y+a x_{0}\right)=A_{2}^{\prime} W(t)\left(y+a x_{0}\right),
$$

proving (2.1). Hence $A_{2}^{\prime} \in \mathcal{G}$. Clearly, $A_{2}, A_{2}^{\prime}$, are not comparable with respect to the operator inclusion, contradicting the hypotheses on $\mathcal{G}$.

"If" is clear.

Proposition 2.11. If $\mathcal{G}$ is finite then there exists $n \in N \cup\{0\}$ such that the cardinality of $\mathcal{G}$ is $2^{n}$.

Proof. Since $\mathcal{G}$ is finite, the codimension of $D(\bar{A})$ in $D(\tilde{A})$ is finite. Assume $\bar{A} \subsetneq \tilde{A}$. Then there exist $n \in N$ and linearly independent elements $x_{1}, x_{2}, \ldots, x_{n}$ in $D(\tilde{A}) \backslash$ $D(\bar{A})$ such that

$$
D(\tilde{A})=D(\bar{A}) \oplus \operatorname{span}\left\{x_{1}, x_{2}, \ldots, x_{n}\right\},
$$

where " $\oplus$ " is the algebraic direct sum. For any subset $\left\{x_{n_{1}}, \ldots, x_{n_{k}}\right\}(1 \leq k \leq n)$ of $\left\{x_{1}, \ldots, x_{n}\right\}$, define

$$
\begin{cases}D(B) & =D(\bar{A}) \oplus \operatorname{span}\left\{x_{n_{1}}, \ldots, x_{n_{k}}\right\}, \text { and } \\ B(y+x) & =\bar{A} y+\tilde{A} x\end{cases}
$$

where $y \in D(\bar{A}), x=a_{1} x_{n_{1}}+\ldots+a_{k} x_{n_{k}}$ for some $a_{j} \in \mathbf{C}(1 \leq j \leq k)$. As with the argument for the closedness of $A_{2}^{\prime}$ in Proposition 2.10, it is easy to show that $B$ is closed by induction. Moreover,

$$
\begin{gathered}
W(t) B \subseteq B W(t) ; \\
B \int_{0}^{t} W(s) x d s=W(t) x-C x, \quad \forall x \in X .
\end{gathered}
$$

$B$ is a subgenerator of $\{W(t)\}_{t \geq 0}$.

Now assume $B \in \mathcal{G}$ and $\bar{A} \subsetneq B$. From (2.3) there exists $\left\{x_{n_{1}}, \ldots, x_{n_{k}}\right\} \subseteq$ $\left\{x_{1}, \ldots, x_{n}\right\}(1 \leq k \leq n)$ such that every $z \in D(B)$ has the decomposition

$$
z=y+x
$$

where $y \in D(\bar{A}), x=a_{1} x_{n_{1}}+\ldots+a_{k} x_{n_{k}}$ for some $a_{j} \in \mathbf{C}(1 \leq j \leq k)$ and $k$ is the minimal positive integer such that (2.5) holds for all $z \in D(B)$. Then

$$
B z=B y+B x=\bar{A} y+\tilde{A} x .
$$

Hence every subset of $\left\{x_{1}, \ldots, x_{n}\right\}$ corresponds to a unique element $B$ in $\mathcal{G}$ defined as in (2.4) and vice versa. In particular, the empty set corresponds to $\bar{A}$ and $\left\{x_{1}, \ldots, x_{n}\right\}$ itself corresponds to $\tilde{A}$. Since the cardinality of the collection of all subsets of $\left\{x_{1}, \ldots, x_{n}\right\}$ is $2^{n}$, that of $\mathcal{G}$ is also.

The following examples show that the set $\mathcal{G}$ of subgenerators of a given $C-$ regularized semigroup may contain $2^{n}$ elements for every $n \in N \cup\{0\}$ or even infinitely many elements.

Example 2.12 ([8]). Let $\mu$ be the Lebesgue measure on $\mathbf{C}$. Define the operator $\tilde{A}$ on $L^{2}(\mathbf{C}, \mu)$ :

$$
(\tilde{A} f)(z)=z f(z) ; \quad D(\tilde{A})=\left\{f \mid f(z), z f(z) \in L^{2}(C, \mu)\right\} .
$$


It is known that $\tilde{A}$ generates the $\exp \left(-|\tilde{A}|^{2}\right)$-regularized semigroup

$$
(W(t) f)(z)=e^{-|z|^{2}} e^{t z} f(z) .
$$

We now prove that the set $\mathcal{G}$ of $\{W(t)\}_{t \geq 0}$ consists of only the element $\tilde{A}$.

Since the set of those $f$ 's in $L^{2}(\mathbf{C}, \mu)$ with compact support is a core for $\tilde{A}$, for every $f \in D(\tilde{A})$, there exists a sequence $\left\{f_{n}\right\}$ in $L^{2}(\mathbf{C}, \mu)$ with compact support such that

$$
\int_{\mathbf{C}}\left|f_{n}(z)-f(z)\right|^{2} d \mu \longrightarrow 0, \int_{\mathbf{C}}|z|^{2}\left|f_{n}(z)-f(z)\right|^{2} d \mu \longrightarrow 0, \text { as } n \longrightarrow \infty .
$$

Write $g_{n}(z)=f_{n}(z) e^{|z|^{2}}$. Then $g_{n} \in D(\tilde{A})$. Introduce $f_{n}(z)=g_{n}(z) e^{-|z|^{2}}$ into (2.6) to conclude that $e^{-|\tilde{A}|^{2}}(D(\tilde{A}))$ is a core for $\tilde{A}$. Since $\tilde{A}$ is densely defined, Corollary 2.9 gives the conclusion.

Example 2.13. Let $X=l^{2}$. For $x=\left(\xi_{1}, \xi_{2}, \ldots\right) \in X$, define

$$
W(t) x=e^{t}\left(0, \xi_{1}, \xi_{2}, \ldots\right) \text { for } t \geq 0 .
$$

Then $\{W(t)\}$ is a $C$-regularized semigroup with

$$
C: x=\left(\xi_{1}, \xi_{2}, \ldots\right) \longrightarrow\left(0, \xi_{1}, \xi_{2}, \ldots\right) .
$$

It is easy to see that $\left\{I, I_{0}\right\}=\mathcal{G}$, where $I$ is the identity on $X$ and $I_{0}$ is the identity on $X_{0} \equiv \operatorname{Im}(C)$.

More generally, let $n \in N$ and define

$$
W(t) x=e^{t}(\underbrace{0, \ldots, 0, \xi_{1}}_{n \text { folds }}, \xi_{2}, \ldots) \quad \forall x=\left(\xi_{1}, \xi_{2}, \ldots\right) \in X .
$$

Then $\{W(t)\}_{t \geq 0}$ is a $C$-regularized semigroup with

$$
C: x=\left(\xi_{1}, \xi_{2}, \ldots\right) \longrightarrow(\underbrace{0, \ldots, 0}_{n \text { folds }}, \xi_{1}, \xi_{2}, \ldots),
$$

and $\mathcal{G}$ contains $2^{n}$ elements.

Example 2.13 shows that there exists a $C$-regularized semigroup so that even if $\tilde{A}(=I)$ is bounded, the set $\mathcal{G}$ of subgenerators may contain more than one element. This is because $C(D(\tilde{A}))\left(=I_{0}(D(\tilde{A}))\right.$ is not a core for $\tilde{A}$.

Example 2.14 ([7]). Let $G \equiv \frac{d}{d x}$, on $X \equiv L^{\infty}(R)$, with maximal domain. Let $A$ be the restriction of $G$ to $D\left(G^{2}\right)$, the domain of $G^{2}$; that is,

$$
D(A) \equiv D\left(G^{2}\right), A x \equiv G x \quad \forall x \in D(A) .
$$

Let $\bar{A}$ be the closure of $A$. Let $C \equiv(1-G)^{-2}$, and define a $C$-regularized semigroup $\{W(t)\}_{t \geq 0}$ by

$$
[W(t) f](s)=(C f)(t+s) \quad(t \geq 0, s \in R) .
$$

Then $G$ is the generator of $\{W(t)\}_{t \geq 0}$. The domain of $\bar{A}$ equals the graph closure of the domain of $G^{2}$, which may be shown to equal $(1-G)^{-1}(B U C(R))$, which does not equal $D(G)=(1-G)^{-1}\left(L^{\infty}(R)\right)$, where BUC(R) is the space of all bounded uniformly continuous functions on $R$. 
Before proceeding further, we define one more regularized semigroup $\left\{W_{1}(t)\right\}_{t \geq 0}$ by (see [7, Example 2.11])

$$
\left[W_{1}(t) f\right](s)=\left[(I-G)^{-1} f\right](t+s) \quad(t \geq 0, s \in R) .
$$

Then $G$ is also the generator of $\left\{W_{1}(t)\right\}_{t \geq 0}$ and for any $f \in X$,

$$
\begin{aligned}
& \frac{1}{t} \int_{s}^{t+s}\left[(I-G)^{-1} f\right](r) d r=\frac{1}{t} \int_{0}^{t}\left[W_{1}(r) f\right](s) d r \\
& \longrightarrow\left[W_{1}(0) f\right](s)=\left[(I-G)^{-1} f\right](s), \text { as } t \longrightarrow 0+,
\end{aligned}
$$

in $X$.

We now prove that $\bar{A}$ is the smallest element in $\mathcal{G}$. Let $A^{\prime} \in \mathcal{G}$. From the boundedness of $G(I-G)^{-1}, A^{\prime}(I-G)^{-2}=G(I-G)^{-2}$ and (2.7),

$$
\begin{gathered}
A^{\prime}\left\{C\left[\frac{1}{t} \int_{s}^{t+s} f(r) d r\right]\right\}=G(I-G)^{-1}\left[\frac{1}{t} \int_{s}^{t+s}\left[(I-G)^{-1} f\right](r) d r\right. \\
\longrightarrow\left[G(I-G)^{-2} f\right](s), \text { as } t \longrightarrow 0+,
\end{gathered}
$$

in $X$. (2.7) also implies that

$$
C\left[\frac{1}{t} \int_{s}^{t+s} f(r) d r\right] \longrightarrow(C f)(s), \text { as } t \longrightarrow 0+,
$$

in $X$. Then $C f \in D\left(A^{\prime}\right)$ for any $f \in X$. This means that $\operatorname{Im}(C) \subseteq D\left(A^{\prime}\right)$, or equivalently, $D(A) \subseteq D\left(A^{\prime}\right)$. Hence $\bar{A} \subseteq A^{\prime} . \bar{A}$ is thus the smallest element in $\mathcal{G}$.

Let $x_{0} \in D(G) \backslash D(\bar{A})$ and define

$$
A_{1}\left(y+a x_{0}\right)=\bar{A} y+a G x_{0} \quad \forall y \in D(\bar{A}), a \in \mathbf{C} .
$$

From the proof of Proposition 2.10, $A_{1}$ is in $\mathcal{G}$. Since there are infinitely many linearly independent choices of $x_{0}, \mathcal{G}$ contains infinitely many elements.

\section{REMARKS}

In this last section, we present several remarks about the $C$-resolvent sets and eigenvalues of subgenerators of a given $C$-regularized semigroup.

Definition 3.1 ([6], [7]). Let $A$ be closed. The complex number $\lambda$ is in $\rho_{c}(A)$, the $C$-resolvent set of $A$, if $\lambda-A$ is injective and $\operatorname{Im}(C) \subseteq \operatorname{Im}(\lambda-A)$.

Lemma 3.2. Let $\lambda \in \mathbf{C}, x \in D(\tilde{A})$, where $\tilde{A}$ is the generator of the $C$-regularized semigroup $\{W(t)\}_{t \geq 0}$. Then $(\lambda-\tilde{A}) x=0$ if and only if $(\lambda-B) C x=0$ for every $B \in \mathcal{G}$.

Proof. Since $\tilde{A}=C^{-1} B C, \tilde{A} x=\lambda x$ if and only if $C^{-1} B C x=\lambda x$ if and only if $B C x=\lambda C x$.

Proposition 3.3. The following are true.

(1) All elements in $\mathcal{G}$ have the same eigenvalues.

(2) Corresponding to the same eigenvalue $\lambda, x$ is an eigenvector of $\tilde{A}$ if and only if $C x$ is an eigenvector of $B$ for every $B \in \mathcal{G}$.

(3) For any $B_{1}, B_{2} \in \mathcal{G}$, if $B_{1} \subseteq B_{2}$ then $\rho_{c}\left(B_{1}\right) \subseteq \rho_{c}\left(B_{2}\right)$. 
Proof. (1),(2) are consequences of Lemma 3.2.

(3) Assume $\lambda \in \rho_{c}\left(B_{1}\right)$. Then $\left(\lambda-B_{1}\right)$ is injective, so $\left(\lambda-B_{2}\right)$ is also by $(1)$. Since $\operatorname{Im}(C) \subseteq \operatorname{Im}\left(\lambda-B_{1}\right) \subseteq \operatorname{Im}\left(\lambda-B_{2}\right)$, we have $\lambda \in \rho_{c}\left(B_{2}\right)$.

It is easy to verify that all results presented in this paper remain valid for sequentially complete locally convex spaces, which appear in [11], [12], and it is also easy to generalize all results obtained in this paper to the case of $n$-times integrated $C$-regularized semigroups (see [12]).

Open questions. The following were proposed by the referee.

(1) When $\operatorname{Im}(C)$ is dense in $X$, are there examples where $\mathcal{G}$ consists of more than one element? Or equivalently, when $\operatorname{Im}(C)$ is dense in $X$, are there examples where $C(D(\tilde{A}))$ is not a core for the generator $\tilde{A}$ ? (See Proposition 2.8(2).)

(2) Proposition 2.8(1) proves that when $\operatorname{Im}(C)$ is dense in $X$, then the minimal element of $\mathcal{G}$ equals the closure of $\tilde{A}$ restricted to $C(D(\tilde{A}))$. Does this remain true in general ?

\section{ACKNOWLEDGEMENT}

The author wishes to highly thank the referee for a painstaking reading of the paper, proposing open questions (1),(2). Proposition 2.8 is, in fact, considered under his motivation.

\section{REFERENCES}

1. W.Arendt, Resolvent positive operators, Proc.London Math.Soc.54(1987), 321-349. MR 88c: 47074

2. W.Arendt, Vector-valued Laplace transforms and Cauchy problems, Israel J. Math. 59(1987), 327-353. MR 89a:47064

3. G. Da Prato, Semigruppi regolarizzabili, Ricerche Mat. 15(1966), 223-248. MR 37:793

4. E.B. Davies and M.M. Pang, The Cauchy Problem and a generalization of the Hille - Yosida theorem, Proc. London Math. Soc. 55(1987), 181-208. MR 88e:34100

5. R. deLaubenfels, Existence and uniqueness families for the abstract Cauchy problem, J. London Math. Soc. 44(1991), 310-338. MR 92k:34075

6. R. deLaubenfels, "Existence families, Functional calculi and Evolution equations", Springer Verlag, Lecture Notes in Math. 1570, 1994. MR 96b:47047

7. R. deLaubenfels, G. Sun and S. Wang, Regularized semigroups, Existence families and the abstract Cauchy problem, J. Diff. and Int. Equ. 8(1995), 1477-1496. MR 96j:47035

8. R. deLaubenfels, C-semigroups and the Cauchy problem, J. Func. Anal. 111 (1993), 44-61. MR 94b: 47055

9. R. deLaubenfels, Z. Huang, S. Wang and Y. Wang, Laplace transforms of polynomially bounded vector-valued functions and semigroups of operators, Israel J. Math., to appear.

10. J.A. Goldstein, "Semigroups of Linear Operators and Applications", Oxford University Press, New York, 1985. MR 87c:47056

11. Y.C. Li, Integrated $C$-semigroups and $C$-cosine functions of operators on locally convex spaces, Ph.D. Dissertation, National Central University, 1991.

12. Y.C. Li and S.Y. Shaw, Integrated $C$-semigroups and the abstract Cauchy problem, preprint 1993.

13. A. Pazy, "Semigroups of Linear Operators and Applications to Partial Differential Equations", Springer, New York, 1983. MR 85g:47061

14. H.R. Thieme, Integrated semigroups and integrated solutions to abstract Cauchy problems, J. Math. Anal. Appl. 152(1990), 416-447. MR 91k:47093

Department of Mathematics, Nanjing University, Nanjing 210093, The People's RePUBLIC OF CHINA

E-mail address: wang2598@netra.nju.edu.cn 